\title{
Combined Expression of Nestin and SPARC Identifies In Situ Tumor Cells in Astrocytic Tumors of all Grades
}

Kifah Aljammal ${ }^{1,2}$, Marie-Françoise Ritz ${ }^{1 *}$, Archana Ramadoss ${ }^{1}$, Guido Sauter ${ }^{3}$, Jean-Louis Boulay ${ }^{1}$ and Luigi Mariani ${ }^{1,2}$

${ }^{1}$ Brain Tumor Biology Laboratory, Department of Biomedicine, University of Basel, Klingelbergstrasse 50, 4056 Basel, Switzerland

${ }^{2}$ Neurosurgery Clinic, Petersgraben 4, 4031 Basel, Switzerland

${ }^{3}$ Institute of Pathology, Universitätsklinikum Hamburg-Eppendorf, Martinistraße 52, 20246 Hamburg, Germany

\section{Abstract}

Background: Malignant gliomas are heterogeneous, diffuse and invasive by nature. Histopathological identification of glioma tumor cells is mandatory to characterize the tumors and the extent of infiltration in the surrounding normal parenchyma.

Methods: In order to identify specific markers for tumor cells not expressed in non-neoplastic brain tissues, we used a well-annotated tissue microarray (TMA) containing 45 samples from patients that suffered from several subtypes of low grade and high grade gliomas (pilocytic astrocytoma, oligoastrocytoma, low grade astrocytoma anaplastic astrocytoma, primary and secondary glioblastomas), non-glial tumors (medulloblastoma and metastasis) as well as fetal, epileptic, and gliotic specimens used as non-tumoral control tissues. The TMA was assessed for 24 proteins involved in tumor proliferation, migration, invasion, and differentiation, or acting as transcription factors and metabolic enzymes.

Results: This immunohistological analysis revealed that nestin and secreted protein, acidic and rich in cysteine (SPARC) are expressed in tumor cells in all glioma subtypes and developmental tissues but rarely in mature epileptic tissue. In addition to these two markers, the expression of mutated isocitrate dehydrogenase 1 (IDH1(R132H)) also identified tumor cells but only in some subtypes of gliomas.

Conclusions: Taken together, our data suggest that the combination of nestin and SPARC expression characterizes tumor glioma cells. These proteins may represent relevant glioma immunohistological markers that might be molecular targets in glioma therapy.

Keywords: Glioma; Tissue microarray; Nestin; IDH1; SPARC

\section{Introduction}

Gliomas are a heterogeneous group of neuroectodermal tumors that arise from the glia. Despite the huge technical development of diagnostic and surgical procedures, the prognosis for patients with gliomas, and particularly glioblastoma multiforme (GBM), remains poor. The survival of these patients does not exceed more than few months (11 to 16 months) after surgical resection and supplemental treatments including chemotherapy and radiation therapy $[1,2]$. Therefore, malignant gliomas are considered as a great challenge for neurosurgery because of their invasive and propagation behaviors, high recurrence rates and substantial morbidity. Low-grade gliomas are usually encountered in younger patient populations between the third and fourth decades of life [3] and most patients survive a decade or more.

Only $30 \%$ of radiologically suspected low-grade gliomas are histopathologically classified as malignant gliomas. Such discrepancies between radiological and histopathological diagnosis indicate that imaging alone is not sufficient in predicting the status of brain tumors. Therefore, a histopathological approach is mandatory in any case of suspected glioma to provide diagnosis, prognosis and prediction of treatment for brain tumors.

Many studies have described over 60 genetic alterations such as mutations, amplifications, and deletions that cause proliferation, infiltration as well as resistance to standard treatments of these tumors [4-6]. The most common changes are amplification of the MDM2, PDGFR, CDK4 genes, mutations of epidermal growth factor receptor (EGFR), isocitrate dehydrogenase 1 (IDH1), PTEN, PIK3CA, and deletion of TP53, RB1, and CDKN2A. Combination of these alterations contributes to the three major pathways PI3K, p53 and RB [7]. There has been considerable interest in the recent years in classifying GBM on the basis of gene expression $[1,8]$. Genetic features can be associated with each type of tumor, but there are still overlaps between groups. For example, in primary GBM, that arises de novo, epithelial growth factor receptor $(E G F R)$ amplification but no IDH1 mutation are described, whereas in secondary GBM, in which pre-existing low-grade glioma was observed, PDGFR amplification, and mutations in IDH1 (on arginine R132), TP53 and RB1 are frequent [9].

Recently, a subpopulation of glioma cells with stem cell characteristics has been described and identified as the tumor initiating cells and the main cause for tumor infiltration, recurrence and therapyresistance $[10,11]$. These tumor cells can be identified by markers specific for stem cells such as the membranous glycoprotein CD133, the transcription factor SOX2 or the cytoskeletal protein nestin. Nestin is down-regulated after development but is re-expressed in the adult organism under certain pathological conditions such as brain injury, ischemia, inflammation and neoplastic transformation [12].

*Corresponding author: Dr. Marie-Françoise Ritz, Tumor Brain Biology Laboratory, Klingelbergstrasse 50, 4056 Basel, Switzerland, Tel: 004161267 15 35; Fax: 00416126716 28; E-mail: marie-francoise.ritz@unibas.ch

Received January 28, 2015; Accepted February 20, 2015; Published February 22, 2015.

Citation: Aljammal K, Ritz MF, Ramadoss A, Sauter G, Boulay JL, et al. (2015) Combined Expression of Nestin and SPARC Identifies In Situ Tumor Cells in Astrocytic Tumors of all Grades. J Cytol Histol 6: 313. doi: 10.4172/2157-7099.1000313

Copyright: (C) 2015 Aljammal K, et al. This is an open-access article distributed under the terms of the Creative Commons Attribution License, which permits unrestricted use, distribution, and reproduction in any medium, provided the original author and source are credited. 
In order to identify specific biomarkers for tumor cells in gliomas, we performed a comparative immunohistological study using a Tissue MicroArray (TMA) containing human tissues isolated from nonneoplastic brains (epileptic, gliotic and fetal brain samples), low grade gliomas (pilocytic astrocytoma (PiA), low grade astrocytoma (LGA), and oligoastrocytoma (OA)), anaplastic astrocytoma (AA), primary GBM (pGBM), secondary GBM (sGBM), and other tumoral tissues (medulloblastoma (Med), and brain metastases (Meta)).

\section{Material and Methods}

\section{Human tissue samples constituting the Tissue MicroArray (TMA)}

All human brain tissue samples were obtained according to the guidelines of the Ethical Committee of Bern, Switzerland, and with informed consent obtained from each patient. The TMA was constructed with paraffin-embedded punched tissue samples $(1 \mathrm{~mm}$ of diameter) from 45 histologically representative cases of different subtypes of gliomas of various WHO grades [13] (LGA: $n=7 ; O A: n=3$; PiA: $n=3$; AA: $n=9$; pGBM: $n=17$; sGBM: $n=6), 10$ non-neoplastic cases (epileptic tissue: $n=4$; fetal tissue: $n=3$; gliotic tissue: $n=3$ ) used as reference control tissues and additional 6 other CNS tumor cases (Med: $\mathrm{n}=5$; Meta: $\mathrm{n}=1$ ). Some tumors were represented by 5 samples forming an invasion gradient ( $3 \mathrm{LGA}, 3 \mathrm{OA}, 3 \mathrm{PiA}, 3 \mathrm{AA}, 8 \mathrm{pGBM}, 3 \mathrm{sGBM}$, $2 \mathrm{Med}$ ), each separated by $2-4 \mathrm{~mm}$ from the center to the periphery of the tumor, in order to visualize potentially infiltrative tumor cells into the surrounding normal appearing brain parenchyma. This TMA was used as a template for immunohistochemistry to identify specific biomarkers for gliomas.

\section{Immunohistochemistry}

The antibodies used for immunohistochemistry are given in Table 1. Prior to incubation with the primary antibody, 2-3 micrometers paraffin-embedded sections were dewaxed, rehydrated, and pretreated as indicated in Table 1. Sections were then (and following all subsequent steps) washed in Tris-buffered saline (TBS) and incubated with the primary antibody diluted in TBS with $0.5 \%$ casein and $5 \%$ normal goat serum, for $60 \mathrm{~min}$ at room temperature. Next, depending on the species in which the primary antibody had been raised, either a 1:300 dilution of a biotinylated goat-anti-mouse immunoglobulin (Ig) or a 1:500 dilution of a biotinylated goat-anti-rabbit Ig antiserum (Dako, Glostrup, Denmark) was applied for $45 \mathrm{~min}$. Thereafter sections were incubated either with an avidin-biotin-complex/horseradish peroxidase (1:100 in TBS) (Vector, Burlingame CA, USA) for $45 \mathrm{~min}$ and developed in $0.02 \% 3,3$ '-diaminobenzidine (DAB) or with 3-amino-9-ethylcarbazole (AEC) (both from Sigma, St. Louis MO, USA) in $0.005 \% \mathrm{H}_{2} \mathrm{O}_{2}$, or with the OptiView DAB detection kit (Benchmark XT Ventana, Tucson, AZ, USA), or with a streptavidin-biotin-complex/alkaline phosphatase (1:200 in TBS) (Dako) for $45 \mathrm{~min}$ and developed with new fuchsin naphtol AS-BI (Sigma, St. Louis MO, USA) for $30 \mathrm{~min}$. Thereafter, all slides were counterstained with hematoxylin, and mounted. Immunostaining without primary antibodies were performed as control in order to verify the specificity of the stainings. No staining was observed in these controls.

\section{Semiquantification of immunopositive signals}

Digital images from the tumor centers were captured with a Leica DMRE microscope set with 40x objective (total magnification 400x) coupled to a ColorView III high resolution CDD color camera, and multiple images (6) were merged in order to reconstitute the whole sample punch section. ImageJ image processing program with manual quantification was used to quantify the total number of cells detected by their nuclei and numbers of cells positive for the specific markers. For nestin and SPARC quantification, positive cells and processes were counted, with the exclusion of the microvessel-associated cells. The number of positive cases/cells was expressed as percentage of total number of cases/cells. In Table 2, $\mathrm{H}+$ represents a number of positive cells $>50 \%$, L+ represents a number of positive cells $<$ or equal to $50 \%$.

\section{Results}

\section{Pre-selection of potential glioma markers from the TMA}

The TMA was assessed for proteins involved in tumor proliferation, migration, invasion, and differentiation, or acting as transcription factors and metabolic enzymes (Figure 1). Throughout the 24 immunohistological stainings some proteins were detected in most cells in all tissue samples including normal non-neoplastic tissues such as PCNA, TN-C, substance P, and MAP2 (Figure 2). These proteins could not be considered specific for tumor cells due to their strong expression in controls tissue samples. In contrast, other proteins were expressed in the tumor samples only, with no or rare immunopositive cells present in non-tumoral control tissues. The quantification of positive cells confirmed that nestin, SPARC and IDH1(R132H) were potential markers for tumor cells. These three markers were then selected for further analysis and quantification in each tumor type present on the TMA (Table 2).

\section{Expression of Nestin, SPARC and IDH1(R132H) in normal brain tissues}

Immunohistochemical staining for nestin revealed numerous nestin-positive cells in fetal tissues (F, about $50 \%$ of the cells, Figure 3 ), but not in epileptic tissues (E), except one single cell (Figure 3, inlet), and the presence of nestin-positive cells associated with the microvasculature (Figure 3, white arrows). Nestin was detected on some reactive astroglial cells in the gliotic tissue ( $G, 15 \%$ of the cells, Figure 3, black arrows), in addition to positive microvascular cells (Figure 3, white arrow). These observations emphasize the expression of nestin under certain circumstances such as proliferation, tissue remodeling (plasticity), or astrocytic activation.

In fetal tissues, SPARC was present in microvessels and was also observed in $25 \%$ of the cells as well as in the extracellular parenchyma (Figure 3, white arrow). Some SPARC-positive cells were observed in epileptic tissues (25\% of cells, Figure 3, black arrows), in addition to some positive cells associated with the microvasculature (Figure 3, white arrow). In gliotic tissue, some rare glial cells expressed SPARC (15\% of the cells, the same cells that are expressing nestin, Figure 3, black arrow), as well as some cells associated with microvessels (Figure 3 , white arrow).

No expression of IDH1(R132H) was observed in these three nontumoral tissue samples (Figure 3).

\section{Expression of Nestin, SPARC and IDH1(R132H) in brain tumors}

Low grade gliomas: Immunohistochemistry revealed the presence of nestin-positive cells in all 13 cases of PiA and OA (in 90\% and 50\% of cells, respectively, Figure 4), and 90\% LGA (Figure 5). In OA, mostly astrocytes expressed nestin, with neoplastic oligodendrocytes showing no staining. Nestin-positive cells were also found in the periphery, as illustrated for the case of OA (Figure $4 \mathrm{p}$ ) and for the 2 cases of LGA (Figure 5p). No sample from the periphery was available for PiA. 


\begin{tabular}{|c|c|c|c|c|c|}
\hline Antibody against & Clone & Source $^{1}$ & Concentration & Pretreatment & Technique $^{2}$ \\
\hline$\beta$-Catenin & 14 & BD Biosciences & $0.6 \mathrm{mg} / \mathrm{ml}$ & Press. cooker, citrate & HRP-DAB \\
\hline bcl-2 & 124 & Dako & $7.5 \mathrm{mg} / \mathrm{ml}$ & Press. cooker, citrate & HRP-DAB \\
\hline bcl-6 & PG-B6p & Dako & $19.5 \mathrm{mg} / \mathrm{ml}$ & Press. cooker, citrate & HRP-DAB \\
\hline c-erbB2 & polyclonal & Dako & $1: 500$ & Press. cooker, citrate & HRP-DAB \\
\hline CNPase & $11-5 B$ & abcam & $1: 1600$ & Microwave, citrate & HRP-DAB \\
\hline Cyclin D1 & P2D11F11 & Novocastra & $6.0 \mathrm{mg} / \mathrm{ml}$ & Microwave, urea & HRP-DAB \\
\hline E-Cadherin & HECD-1 & R\&D Systems & $1.0 \mathrm{mg} / \mathrm{ml}$ & Press. cooker, citrate & AP-NF \\
\hline EGFR & $5 \mathrm{~B} 7$ & Ventana & Ready to use & Pronase & HRP-DAB \\
\hline GFAP & $6 \mathrm{~F} 2$ & Dako & $2.6 \mathrm{mg} / \mathrm{ml}$ & Microwave, citrate & HRP-DAB \\
\hline Hey1 & polyclonal & abcam & $1: 500$ & No pretreatment & HRP-AEC \\
\hline IDH1(R132H) & $\mathrm{H} 09$ & dianova & $1: 20$ & Microwave, citrate & HRP-DAB \\
\hline MAP2 & $\mathrm{HM}-2$ & Sigma-Aldrich & $1: 20000$ & Tris-EDTA pH 8.5 & HRP-DAB \\
\hline MIB1 & MIB1 & Dako & $1.6 \mathrm{mg} / \mathrm{ml}$ & Press. cooker, citrate & HRP-DAB \\
\hline Nestin & $10 \mathrm{C} 2$ & Serotec & $1: 50$ & Tris-EDTA pH 8.5 & HRP-DAB \\
\hline Neurofilament & $2 \mathrm{~F} 11$ & Dako & $5.4 \mathrm{mg} / \mathrm{ml}$ & Press. cooker, citrate & AP-NF \\
\hline Osteonectin & $15 G 12$ & Leica & $1: 500$ & Microwave, citrate & HRP-DAB \\
\hline p53 & DO-7 & Dako & $1.0 \mathrm{mg} / \mathrm{ml}$ & Press. cooker, citrate & HRP-DAB \\
\hline PCNA & PC10 & Dako & $7.7 \mathrm{mg} / \mathrm{ml}$ & Microwave, citrate & HRP-DAB \\
\hline Sox2 & $6 \mathrm{~F} 1.2$ & Millipore & $1: 100$ & No pretreatment & HRP-AEC \\
\hline STAT3- $\alpha$ & polyclonal & Cell Signaling & $1: 200$ & Microwave, urea & HRP-DAB \\
\hline Substance P & polyclonal & BioGenex & $1: 1600$ & Microwave, citrate & HRP-DAB \\
\hline Synaptophysin & Snp88 & BioGenex & $1: 100$ & Press. cooker, citrate & AP-NF \\
\hline Tenascin C & 49 & Leica & $1: 50$ & Microwave, citrate+trypsin & HRP-DAB \\
\hline Vimentin & 3B4 & Dako & $0.5 \mathrm{mg} / \mathrm{ml}$ & Trypsin & HRP-DAB \\
\hline \multicolumn{6}{|c|}{$\begin{array}{l}\text { 'Manufacturer locations: abcam, Cambridge, UK; BD Biosciences, Basel, Switzerland; BioGenex, San Ramon, CA, USA; Cell Signaling, Beverly, MA, USA; Dako, } \\
\text { Glostrup, Denmark; Dianova, Hamburg, Germany; Leica, Nünningen, Switzerland; Millipore, Merck Millipore, Darmstadt, Germany; Novocastra, Newcastle-upon- } \\
\text { Tyne, UK; R\&D Systems, Minneapolis, MN, USA; Serotec, Düsseldorf, Germany; Sigma-Aldrich, St-Louis, MO, USA; Ventana Medical Systems, Tucson, AZ, USA. } \\
\text { echniques: AP-NF: ABC method using alkaline phosphatase and new fuchsin; AEC: 3-amino-9-ethylcarbazole; HRP-DAB: ABC method using horseradish peroxidase } \\
\text { and 3,3'-diaminobenzidine (DAB) or OptiView DAB detection kit/Benchmark XT (Ventana Medical Systems). }\end{array}$} \\
\hline
\end{tabular}

Table 1: Primary antibodies used for immunohistochemistry and technical information.

\begin{tabular}{|c|c|c|c|c|c|c|c|c|c|c|c|c|c|}
\hline \multicolumn{6}{|c|}{ Nestin } & \multicolumn{4}{|c|}{ SPARC } & \multicolumn{4}{|c|}{ IDH1(R132H) } \\
\hline Tissue & $n$ & $\mathrm{H}+$ & L+ & $\mathrm{Neg}$ & $\%+$ & $\mathrm{H}+$ & $\mathrm{L}+$ & Neg & $\%+$ & $\mathrm{H}+$ & $\mathrm{L}+$ & Neg & $\%+$ \\
\hline $\mathrm{F}$ & 3 & - & 3 & - & 100 & - & 3 & - & 100 & - & - & 3 & 0 \\
\hline$E$ & 4 & - & - & 4 & 0 & - & 4 & - & 100 & - & - & 4 & 0 \\
\hline G & 3 & - & 3 & - & 100 & - & 3 & - & 100 & - & - & 3 & 0 \\
\hline PiA & 3 & 3 & - & - & 100 & 3 & - & - & 100 & - & - & 3 & 0 \\
\hline $\mathrm{OA}$ & 3 & - & 3 & - & 100 & 2 & 1 & - & 100 & 3 & - & - & 100 \\
\hline LGA & 7 & 5 & 2 & - & 100 & 5 & 2 & - & 100 & 5 & - & 2 & 71 \\
\hline AA & 9 & 8 & 1 & - & 100 & 8 & 1 & - & 100 & 6 & 1 & 2 & 77 \\
\hline pGBM & 17 & 13 & 4 & - & 100 & 10 & 7 & - & 100 & - & - & 17 & 0 \\
\hline sGBM & 6 & 3 & 3 & - & 100 & 2 & 2 & 2 & 66 & 4 & 2 & - & 100 \\
\hline Med & 5 & - & 5 & - & 100 & 3 & 2 & - & 100 & - & - & 5 & 0 \\
\hline Meta & 1 & - & 1 & - & 100 & - & 1 & - & 100 & - & - & 0 & 0 \\
\hline
\end{tabular}

Abbreviations: F: fetal tissue; E: epilepsy tissue; G: gliotic tissue; PiA: pilocytic astrocytoma; OA: oligoastrocytoma; LGA: Low-grade astrocytoma; AA; anaplastic astrocytoma; pGBM: primary glioblastoma; sGBM: secondary glioblastoma; Med: medulloblastoma; Meta; metastasis. $\mathrm{n}$ : number of cases, $\mathrm{H}+$ : $>50 \%$ of positive cells; L+: < or equal to $50 \%$ positive cells; Neg: negative, no positive cells; $\%+$ : percentage of positive cases.

Table 2: Number of positive cases for nestin, SPARC and IDH1(R132H) in the different control brain samples and the brain tumor samples.

Similar observations were done for SPARC. SPARC-positive cells were present in all cases of low grade glioma (PiA, OA and LGA), with about $90 \%$ of positive cells in the tumor center but also in the periphery of the tumor (Figure 4 and $5 c$ and $5 p$ ).

IDH1(R132H) immunopositivity was found in none of the PiA cases, in $75 \%$ of cells in all 3 cases of OA (Figure 4 and Table 2), and in $90 \%$ of cells in 5 out of 7 cases of LGA (Figure 5 and Table 2). In OA and the positive cases of LGA, IDH1(R132H)-positive cells were also present in the periphery (Figure $4 p$ and 5 case 1,5p).

Anaplastic astrocytoma: IHC analysis of AA showed nestin, as well as SPARC expressed in 80 - to $90 \%$ of cells in most of the cases analysed, whereas 7 out of 9 cases were IDH1(R132H) immunopositive (Figure 6 and Table 2). Cells positive for all the three markers were found also in the periphery of the tumors (Figure 6, case 1, 6p). Interestingly, in IDH1(R132H)-negative cases, Nestin and SPARC were also expressed by cells in the infiltration zone (Figure 6, case 2, 6p), suggesting that their expressions are independent of IDH1 $(\mathrm{R} 132 \mathrm{H})$ status. Histopathological examination supports that the expression of both proteins is specific for tumor cells, in which case, both markers may be reliable for recognizing IDH1(R132H)-negative tumors. 


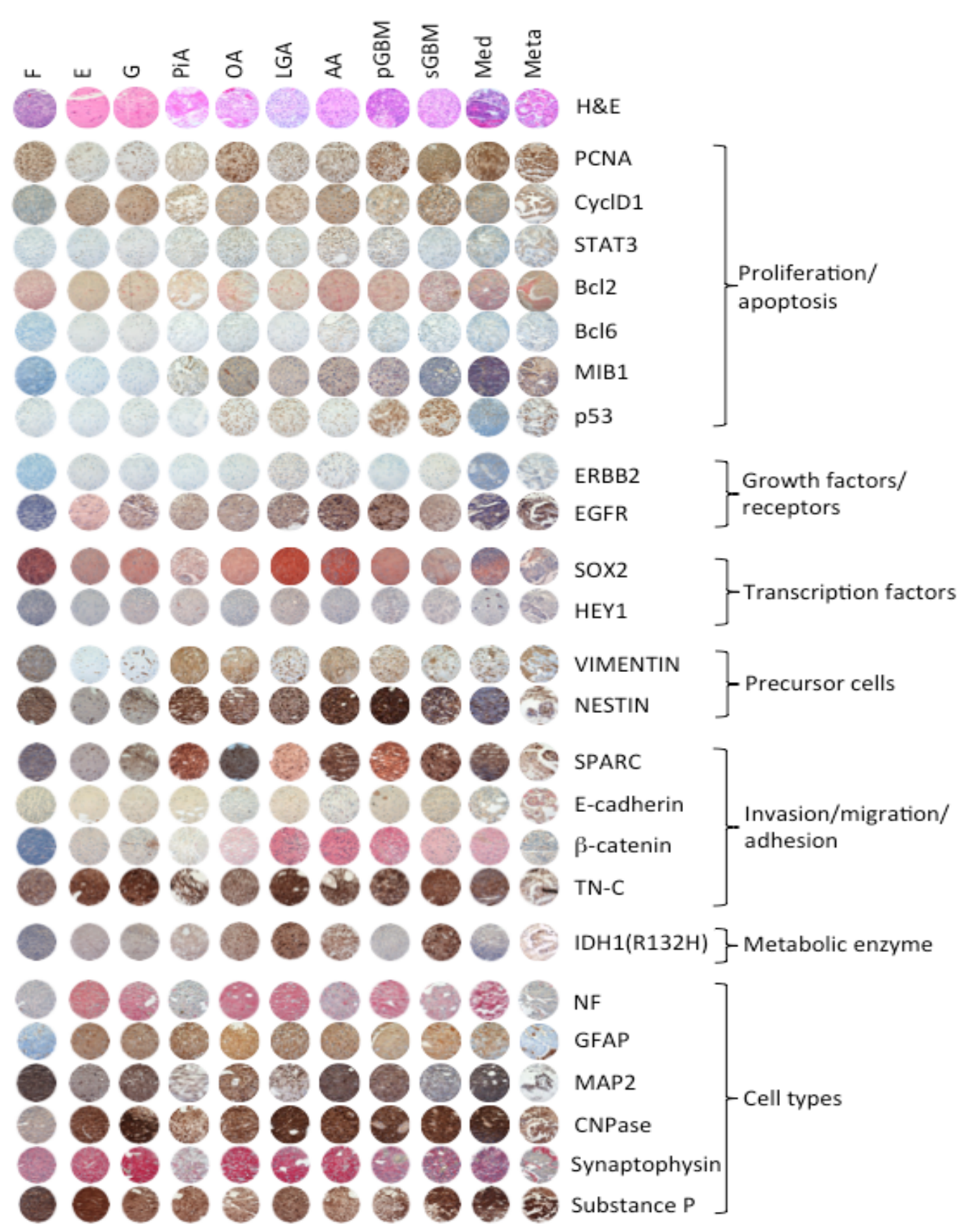

Figure 1: Overview of representative punches of control and tumor tissue samples stained with Hematoxylin/Eosin (H/E) or immunostained for the 24 specific proteins grouped by categories.

Primary GBM: Nestin-positive cells, as well as SPARC-positive cells ( $90 \%$ of cells for both markers) have been detected in all 17 cases of pGBM (Table 2). SPARC showed intra-and extracellular expression profiles in the center of the tumor (Figure 7, upper part, c). Of note, none of these pGBM showed IDH1(R132H) expression. The expressions of Nestin and SPARC were very strong and delineated clear margins between the tumor mass and the infiltration zone, however, a small number of cells expressing Nestin and SPARC were also observed in the periphery of the tumors (Figure 7, upper part, p).

Secondary GBM: Out of the 6 sGBM cases, we observed strong staining for nestin in 3 cases, in 2 cases for SPARC ( 80 to $90 \%$ of the cells), as well as in 4 cases for IDH1(R132H) (90\% of cells) (Figure 7 , lower part, c). In these cases, nestin, SPARC and IDH1(R132H) were also expressed in cells in the infiltration zone of the tumors (Figure 7, lower part, p).

Two cases did not express SPARC in the tumor, or only in very rare astrocytes. Out of these 2 cases, one showed a low expression of Nestin (case 1, Figure 8), and the other strongly expressed nestin (case 2 in Figure 8). IDH1(R132H) was strongly expressed in both cases (Figure 8). In these 2 cases, a high cellularity was observed, and cells were expressing the proliferative cell nuclear antigen (PCNA), and 

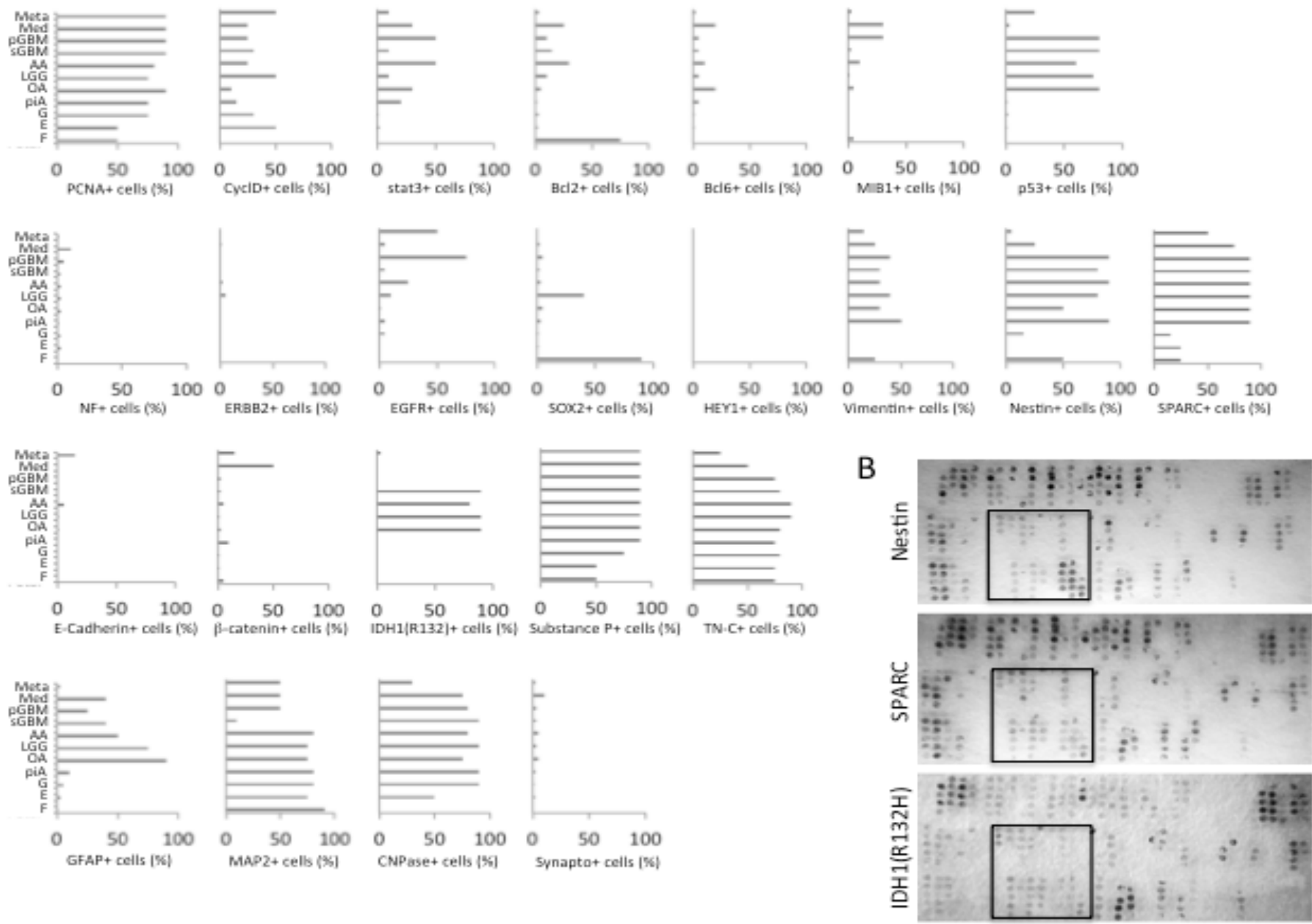

Figure 2: Quantitative analysis of each individual marker. A: Quantification of positive cells for each marker in control and tumor tissue samples, expressed as \% of the total number of cells in the region of interest. Three markers were highly expressed in most of gliomas and absent in normal controls and less frequent in other tumors: nestin, SPARC and IDH1(R132H). B: General views of the TMA showing tissue samples represented by 5 sections each (when available). The comparison of the staining of the three selected markers shows co-expression of nestin and SPARC in numerous samples, whereas a smaller number of samples express IDH1(R132H). The non-tumoral control cases (F, E and $\mathrm{G})$ are located in the frames.

p53, reflecting the presence of loss-of-function mutation of this antitumoral protein (Figure 8). Images from case 2 probably represent the so-called "hot-spots" of proliferative cells. Considering the great morphologic heterogeneity of GBM, it is possible that the samples taken from case 2 hit such hot spots of IDH1(R132H)-positive tumor cells that clearly express nestin, as well as proliferation markers, but not SPARC, indicated by the black arrows in Figure 8. This suggests also that highly proliferative tumor cells do not express SPARC, and that this marker may be expressed during an earlier or later phase of tumor progression.

\section{Expression of Nestin, SPARC and IDH1(R132H) in other tumor types}

Medulloblastoma: Almost no IDH1(R132H) staining was observed in medulloblastoma samples, but they all show SPARC expression, 3 cases in more than $75 \%$ of the cells, and 2 showing lower expression (Figure 9, upper part). Moreover, extracellular SPARC was also found in the parenchyma in all high positive cases. In contrast to gliomas, nestin-positive cells were mainly observed in the tumor vasculature, and only some isolated cells expressed nestin in the parenchyma $(25 \%$ of cells) in all cases.
Metastasis: In the single case of brain metastasis, immunolabeling of pseudo-rosettes surrounding blood vessels were observed (Figure 9, lower part). Nestin was strongly expressed by endothelial cells, but tumor cells constituting the rosettes showed less intense nestin immunoreactivity, and some nestin-positive tumor cells outside the cell clusters were also observed ( $25 \%$ of cells). In this metastasis, $50 \%$ of cells strongly expressed SPARC. This protein was also expressed in vascular cells located at the center of the rosettes. All the cells in the tumor were IDH1(R132H) negative.

\section{Discussion}

Among the 24 proteins stained in our TMA, IDH1(R132H), nestin and SPARC were clustered in most primary and secondary brain tumors. They were then further analysed in order to identify their potential as specific markers for tumor cells. These markers were concentrated in the center of the tumors, and expressed also by tumor cells infiltrating normal tissue.

Nestin belongs to class VI intermediate filaments and is expressed in proliferating cells during the developmental stages in a variety of embryonic and fetal tissues. During development, nestin is produced 
Citation: Aljammal K, Ritz MF, Ramadoss A, Sauter G, Boulay JL, et al. (2015) Combined Expression of Nestin and SPARC Identifies In Situ Tumor Cells in Astrocytic Tumors of all Grades. J Cytol Histol 6: 313. doi:10.4172/2157-7099.1000313

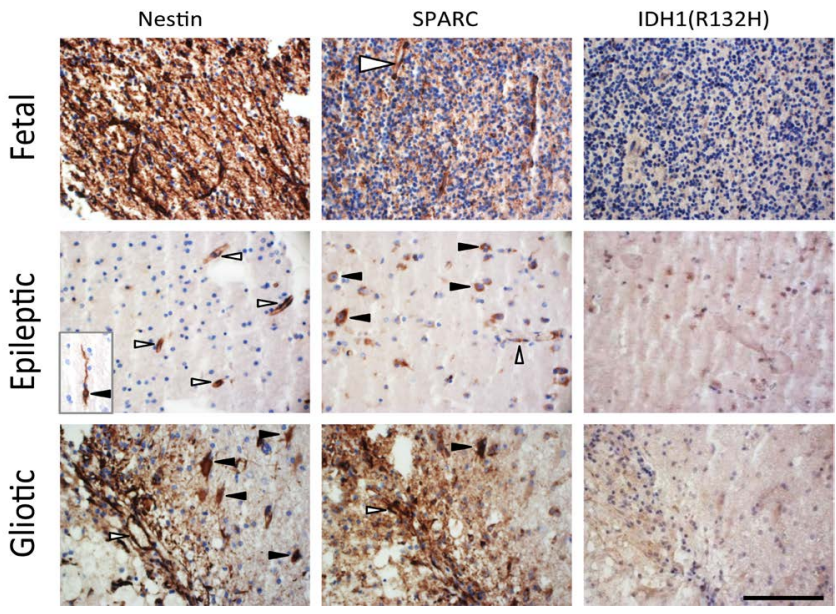

Figure 3: Representative micrographs of fetal, epileptic and gliotic tissues immunostained for nestin, SPARC and IDH1(R132H). IDH1(R132H) was not expressed in these control brain samples, whereas nestin was highly expressed in fetal tissue, not in epilepsy (except one single cell shown in inlet), but was present in reactive glial cells in gliotic tissue (black arrows) and in microvessels (white arrows). SPARC was expressed in reactive glial cells in gliotic tissue (black arrows), as well as in rare cells in epileptic tissue (black arrows), in addition to some vascular cells (white arrows). Original magnification $\times 400$ Scale bar: $100 \mu \mathrm{m}$
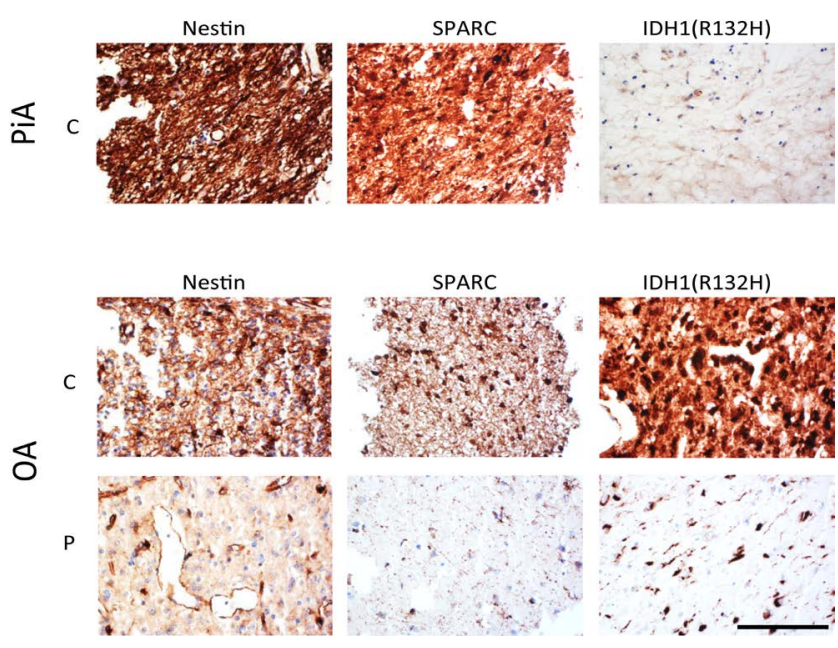

Figure 4: Representative micrographs of pilocytic astrocytoma $(\mathrm{PiA})$ and oligoastrocytoma (OA) immunostained for nestin, SPARC and IDH1(R132H). The $\mathrm{PiA}$ was IDH1(R132H) negative. The case of OA was positive for all three markers. Center of the tumor (c) of the PiA and the OA, as well as the periphery (p) of the OA are depicted. Original magnification $\times 400$. Scale bar: $100 \mu \mathrm{m}$.

in neuronal and glial stem/progenitor cells in the mammalian CNS and is consecutively replaced by other intermediate filament proteins (neurofilaments or GFAP). Nestin has also been shown to be expressed in other cell types such as endothelial cells of capillaries [14], smooth muscle cells and pericytes in the CNS capillaries $[15,16]$, but not in larger blood vessels. This is in accordance with our observations that most of the capillaries were nestin-positive, even in the normal, nontumoral tissues. Interestingly, a rapid re-expression of nestin was found in reactive astrocytes of the adult rodent brain after seizures [17], trauma [18] and brain ischemia [19,20], reflecting the reversal of astrocytes to a more immature phenotype during the reactive state. We observed only one single cell expressing nestin in our epilepsy samples that may constitute a glia cell involved in plasticity.
Nestin expression is used as a marker of proliferating and migrating cells, as well as in brain tumors including GBMs [21,22]. Indeed, all tumor cases analysed in the present study, including medulloblastoma and metastasis, showed various levels of cells expressing nestin. The higher expression level of nestin in gliomas of astrocytic lineage $(90 \%$ of tumor cells in PiA, LGA, AA) compared to OA (only $50 \%$ of tumor cells) illustrates the specificity of nestin for astrocytoma cells and not for oligodendrocytes. In astrocytic gliomas, an association between

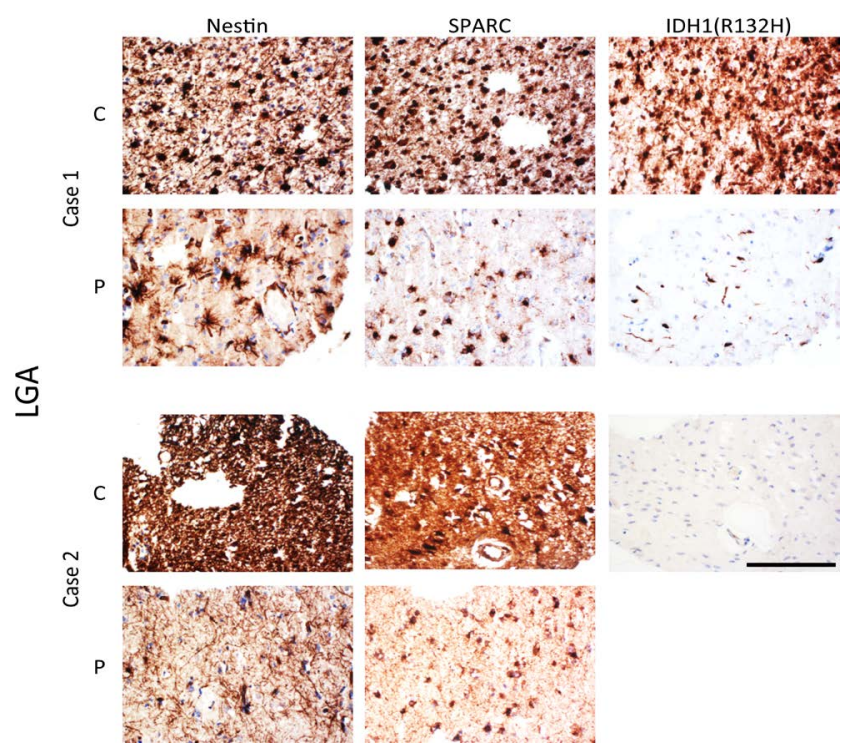

Figure 5: Representative micrographs of two cases of low grade astrocytomas immunostained for nestin, SPARC and IDH1(R132H). Case 1 expressed the mutated IDH1 protein, whereas case 2 was clearly IDH1(R132H)-negative. Both cases were nestin and SPARC immunopositive. The center (c) and periphery $(p)$ of the corresponding tumors are depicted for each case. Original magnification $\times 400$. Scale bar: $100 \mu \mathrm{m}$.

$\varangle$
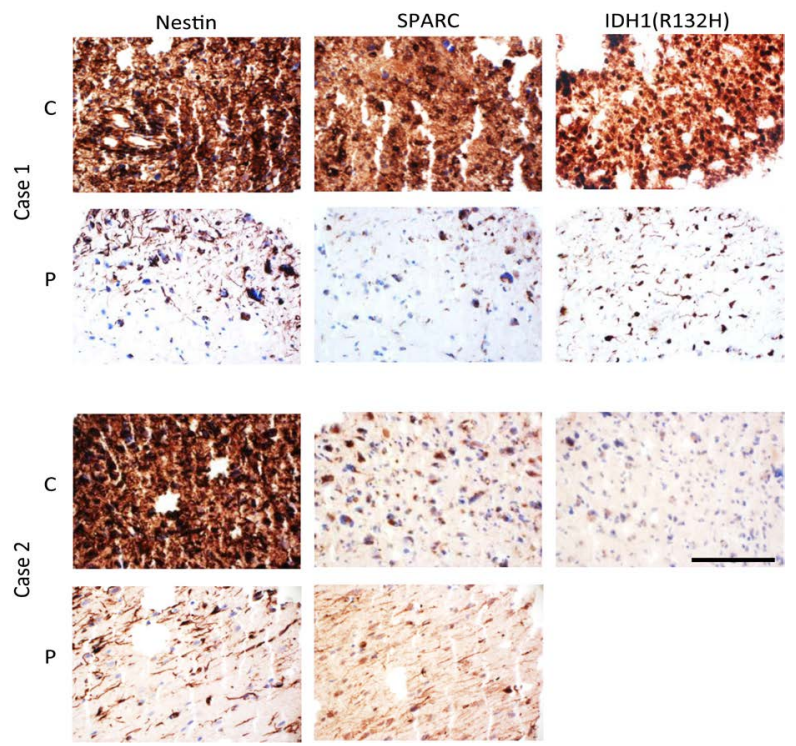

Figure 6: Representative micrographs of two cases of anaplastic astrocytoma immunostained for nestin, SPARC and IDH1(R132H). Case 1 was positive for all three markers, whereas case 2 was IDH1(R132H) negative and low for SPARC expression. For both cases, the center (c) and periphery $(p)$ of the tumors are depicted. Original magnification $\times 400$. Scale bar: $100 \mu \mathrm{m}$. 

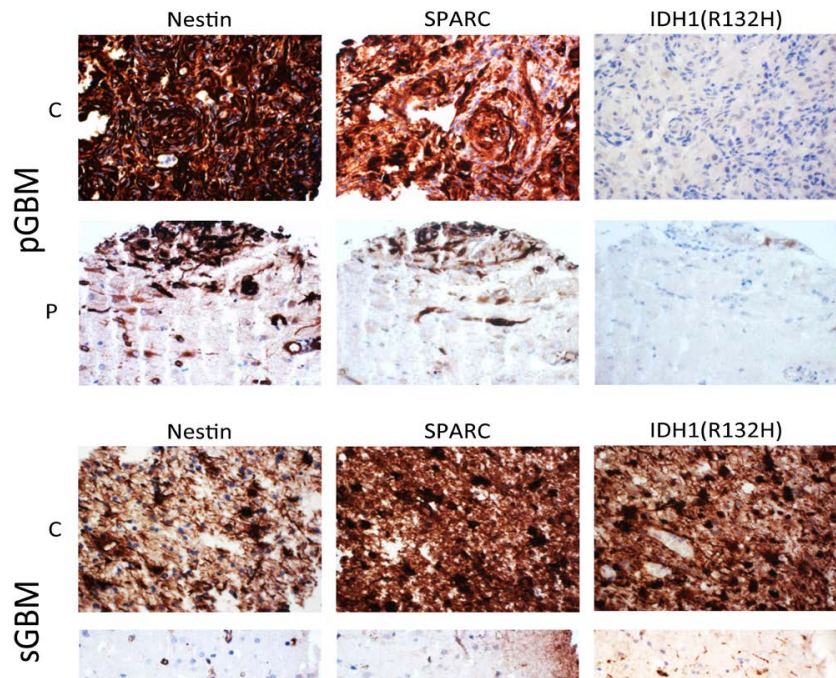

$P$
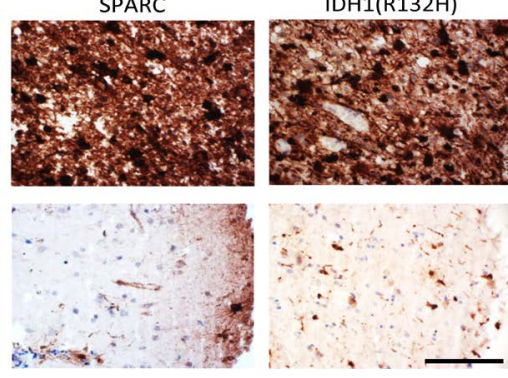

Figure 7: Representative micrographs of tumors in $\mathrm{pGBM}$ and $\mathrm{sGBM}$ immunostained for nestin, SPARC and IDH1(R132H). The case of sGBM was positive for all three markers, whereas pGBM was IDH1(R132H) negative. The center $(c)$ and periphery $(p)$ of the tumors are shown. Original magnification $\mathrm{x} 400$. Scale bar: $100 \mu \mathrm{m}$.

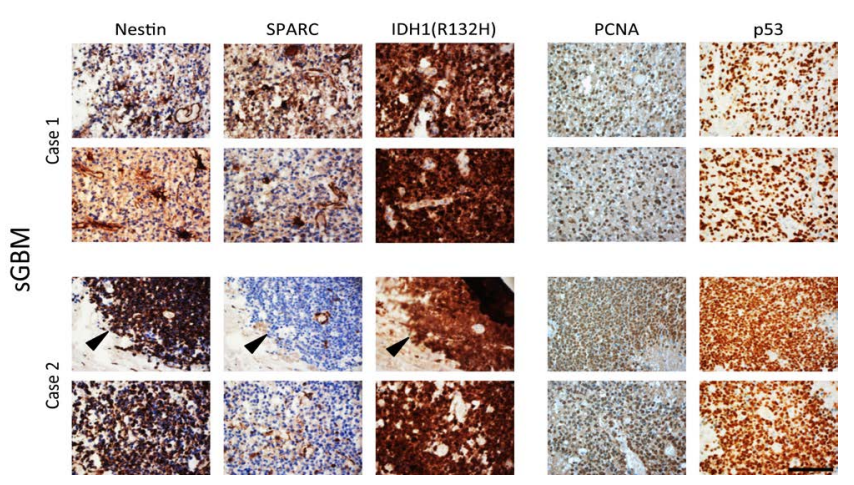

Figure 8: Representative micrographs of the tumor centers of two cases of SGBM with low SPARC immunostaining. The two cases (case 1 and 2) showed high cellularity, with strong nuclear expression of PCNA and p53 in almost all cells, in addition to the expression of IDH1(R132H). Case 1 showed low number of nestin-positive cells, whereas case 2 revealed strong nestin immunostaining in all cells at the "hot spots" indicated by black arrowheads. Original magnification $\times 400$. Scale bar: $100 \mu \mathrm{m}$.

the expression of transcription repressors, the nuclear receptor corepressors (NCORs) involved in maintaining undifferentiated and proliferating state, and nestin has been observed, supporting a role of the later in this type of gliomas [23].

Very recently, nestin has been shown to be expressed also at the cell surface of human glioma stem cells [24]. It is suggested, therefore, that this cell surface-associated nestin may serve as a promising glioma stem cell marker.

We observed nestin expressed in cell processes in infiltrated tissue, suggesting that nestin might reflect the limits of the tumor mass but also single infiltrating tumor cells.
Secreted SPARC regulates extracellular matrix, and matrix metalloproteinases activity, modulates cell growth, cell morphology and migration responses [25-27]. Intracellular SPARC, however, might have different functions in glioma, such as differentiation and survival [28]. Through the CNS, SPARC is highly localized in mature ramified microglia. Following ischemic and excitotoxic injury, reactive perilesional astrocytes upregulate the expression of SPARC [29]. Accordingly, we found reactive astrocytes expressing intracellular SPARC in gliotic samples.

Interestingly, SPARC has been shown to induce cell cycle arrest in tumor cells $[30,31]$ and to functionally contribute to glioma invasion in vitro and in vivo [32-34]. Indeed, it is well known that migration and proliferation are antagonistic cellular behaviors. In a model of glioma cells xenografts, the patterns of invasion and the extent of growth delay correlated with the level of SPARC expressed. It has been proposed therefore that the ability of SPARC to promote invasion depends on the level of its secretion and the resultant modulation of the level of adherence and motility induced [32]. We observed no SPARC in highly proliferating stem-like cells located at proliferation "hot spots" in 2 cases of sGBM, but the presence of extracellular SPARC in numerous cases of fetal tissue and in gliomas, in which remodeling and invasion are active. Therefore, this observation is in accordance with the "go or growth" theory illustrating the fact that factors promoting glioma invasion delay cell cycle and tumor growth. Therefore, targeting SPARC may represent an anti-invasion therapeutic way for malignant gliomas, together with an anti-proliferative agent.

IDH1 mutations are the earliest detectable genetic alterations in low-grade diffuse astrocytomas and oligodendrogliomas, suggesting that the developmental pathways of these tumors are distinct from those of pGBM. Secondary GBMs progress from precursor lowgrade diffuse astrocytoma or anaplastic astrocytoma. Histologically, primary and secondary GBMs are largely indistinguishable, but they differ in their genetic and epigenetic profiles. Our results confirm that IDH1(R132H) mutation recognizes most LGG and resulting sGBMs, whereas it is strictly absent in pGBMs [35].

Taken together, our results indicate that nestin and SPARC are coexpressed in most brain tumor cells, highlighting the full extent of the tumors: the core and the infiltration zone. Nestin is expressed in all brain tumors, particularly in cell populations with robust proliferative, clonogenic and tumorigenic capacity, supporting its potential value as marker for neoplastic cells. This finding might be helpful to detect tumor cell infiltrations in the peripheral margin of gliomas, eventually also in cell processes. In contrast, SPARC is expressed also in nontumoral tissue, but is low in some sGBM, in which active tumor cell proliferation at the "hot spots" is observed. Moreover, its presence in normal cerebral samples such as epileptic tissue makes SPARC a less specific marker than nestin.

IDH1(R132H) is the gold-standard marker for the diagnosis of mutation-bearing gliomas, and represents the most sensitive marker differentiating $\mathrm{pGBM}$ from sGBM. We suggest that these three proteins, nestin, SPARC and IDH1 $(\mathrm{R} 132 \mathrm{H})$, taken together, are potential markers for brain tumor cells. The need for tumor specifictargeted therapies is one major therapeutic challenge for the future. The understanding of the molecular pathways and genetic complexity of various subtypes of gliomas has increased the number of novel glioma therapeutic strategies, such as gene therapy, stem cell-based or nanoparticle-based therapies [36]. However, the identification of common markers specific for all gliomas and other brain tumors such as medulloblastoma may simplify the development of a single 


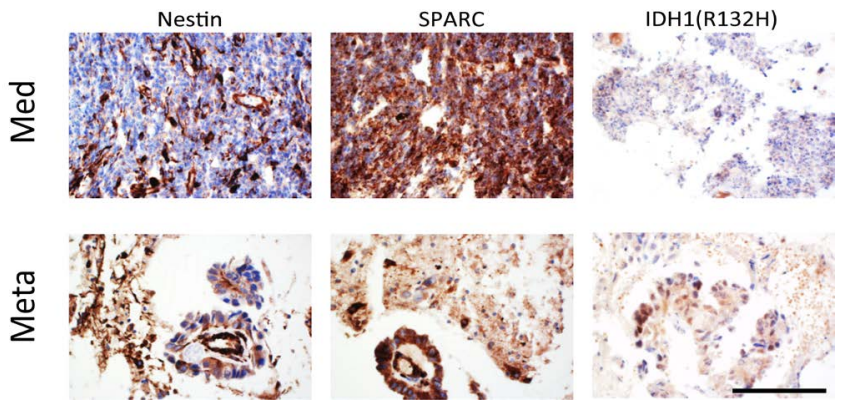

Figure 9: Representative micrographs of medulloblastoma and metastasis immunostained for nestin, SPARC and IDH1(R132H). Nestin was mainly observed expressed by tumor vasculature and some rare tumor cells. SPARC expression was strong in medulloblastoma cells and the cells constituting the rosette in the metastasis. No IDH1 $(\mathrm{R} 132 \mathrm{H})$ staining was observed in these two cases. Original magnification $\times 400$. Scale bar: $100 \mu \mathrm{m}$.

therapeutic approach in glioma treatment. In this regard, nestin and SPARC may constitute potential brain tumor biomarkers.

\section{Acknowledgments}

We thank Prof. Stephan Frank, Institute of Neuropathology Basel for neuropathological descriptions of the cases, David Friedrich and Monika Schobesberger for their technical assistance. We would like to warmly acknowledge the assistance of Dr. Andreas Kappeler, Institute of Neuropathology Bern, and Markus Bänziger, Institute of Neuropathology Basel, for help with the $\mathrm{IHC}$ protocols.

\section{References}

1. Verhaak RG, Hoadley KA, Purdom E, Wang V, Qi Y, et al. (2010) Integrated genomic analysis identifies clinically relevant subtypes of glioblastoma characterized by abnormalities in PDGFRA, IDH1, EGFR, and NF1. Cancer Cell 17: 98-110.

2. Stupp R, Dietrich PY, Ostermann Kraljevic S, Pica A, Maillard I, et al. (2002) Promising survival for patients with newly diagnosed glioblastoma multiforme treated with concomitant radiation plus temozolomide followed by adjuvant temozolomide. J Clin Oncol 20: 1375-1382.

3. Pouratian N, Schiff D (2010) Management of low-grade glioma. Curr Neurol Neurosci Rep 10: 224-231.

4. Belden CJ, Valdes PA, Ran C, Pastel DA, Harris BT, et al. (2011) Genetics of glioblastoma: a window into its imaging and histopathologic variability. Radiographics 31: 1717-1740.

5. Parsons DW, Jones S, Zhang X, Lin JC, Leary RJ, et al. (2008) An integrated genomic analysis of human glioblastoma multiforme. Science 321: 1807-1812.

6. Appin CL, Brat DJ (2014) Molecular genetics of gliomas. Cancer J 20: 66-72.

7. The Cancer Genome Atlas (TCGA) Research Network (2008) Comprehensive genomic characterization defines human glioblastoma genes and core pathways. Nature 455: 1061-1068.

8. Dunn GP, Rinne ML, Wykosky J, Genovese G, Quayle SN, et al. (2012) Emerging insights into the molecular and cellular basis of glioblastoma. Genes Dev 26: 756-784.

9. Watanabe T, Nobusawa S, Kleihues P, Ohgaki H (2009) IDH1 mutations are early events in the development of astrocytomas and oligodendrogliomas. Am J Pathol 174: 1149-1153.

10. Singh S, Dwarakanath BS, Mathew TL (2004) DNA ligand Hoechst-33342 enhances UV induced cytotoxicity in human glioma cell lines. J Photochem Photobiol B 77: 45-54.

11. Johannessen TC, Bjerkvig R, Tysnes BB (2008) DNA repair and cancer stemlike cells--potential partners in glioma drug resistance? Cancer Treat Rev 34: 558-567

12. Veselska R, Kuglik $P$, Cejpek $P$, Svachova $H$, Neradil J, et al. (2006) Nestin expression in the cell lines derived from glioblastoma multiforme. BMC Cancer 6: 32.
13. Louis DN, Ohgaki H, Wiestler OD, Cavenee WK, Burger PC, et al. (2007) The 2007 WHO classification of tumours of the central nervous system. Acta Neuropathol 114: 97-109.

14. Klein T, Ling Z, Heimberg H, Madsen OD, Heller RS, et al. (2003) Nestin is expressed in vascular endothelial cells in the adult human pancreas. $J$ Histochem Cytochem 51: 697-706.

15. Alliot F, Rutin J, Leenen PJ, Pessac B (1999) Pericytes and periendothelial cells of brain parenchyma vessels co-express aminopeptidase $\mathrm{N}$, aminopeptidase A, and nestin. J Neurosci Res 58: 367-378.

16. Dore-Duffy P, Katychev A, Wang X, Van Buren E (2006) CNS microvascular pericytes exhibit multipotential stem cell activity. J Cereb Blood Flow Metab 26: 613-624.

17. Clarke SR, Shetty AK, Bradley JL, Turner DA (1994) Reactive astrocytes express the embryonic intermediate neurofilament nestin. Neuroreport 5: 1885-1888.

18. Holmin S, Schalling M, Höjeberg B, Nordqvist AC, Skeftruna AK, et al. (1997) Delayed cytokine expression in rat brain following experimental contusion. $\mathrm{J}$ Neurosurg 86: 493-504.

19. Chopp M, Li Y, Jiang N (1999) Increase in apoptosis and concomitant reduction of ischemic lesion volume and evidence for synaptogenesis after transient focal cerebral ischemia in rat treated with staurosporine. Brain Res 828: 197-201.

20. Schmidt-Kastner R, Bedard A, Hakim A (1997) Transient expression of GAP43 within the hippocampus after global brain ischemia in rat. Cell Tissue Res 288: 225-238.

21. Ehrmann J, Kolár Z, Mokry J (2005) Nestin as a diagnostic and prognostic marker: immunohistochemical analysis of its expression in different tumours. $J$ Clin Pathol 58: 222-223.

22. Schiffer D, Manazza A, Tamagno I (2006) Nestin expression in neuroepithelia tumors. Neurosci Lett 400: 80-85.

23. Campos B, Bermejo JL, Han L, Felsberg J, Ahmadi R, et al. (2011) Expression of nuclear receptor corepressors and class I histone deacetylases in astrocytic gliomas. Cancer Sci 102: 387-392.

24. Jin X, Jin X, Jung JE, Beck S, Kim H (2013) Cell surface Nestin is a biomarker for glioma stem cells. Biochem Biophys Res Commun 433: 496-501.

25. Barker TH, Baneyx G, Cardó-Vila M, Workman GA, Weaver M, et al. (2005) SPARC regulates extracellular matrix organization through its modulation of integrin-linked kinase activity. J Biol Chem 280: 36483-36493.

26. Rempel SA, Golembieski WA, Fisher JL, Maile M, Nakeff A (2001) SPARC modulates cell growth, attachment and migration of U87 glioma cells on brain extracellular matrix proteins. J Neurooncol 53: 149-160.

27. Said N, Najwer I, Motamed K (2007) Secreted protein acidic and rich in cysteine (SPARC) inhibits integrin-mediated adhesion and growth factor-dependent survival signaling in ovarian cancer. Am J Pathol 170: 1054-1063.

28. Shi Q, Bao S, Song L, Wu Q, Bigner DD, et al. (2007) Targeting SPARC expression decreases glioma cellular survival and invasion associated with reduced activities of FAK and ILK kinases. Oncogene 26: 4084-4094.

29. Lloyd-Burton SM, York EM, Anwar MA, Vincent AJ, Roskams AJ (2013) SPARC regulates microgliosis and functional recovery following cortical ischemia. J Neurosci 33: 4468-4481.

30. Chetty C, Dontula R, Ganji PN, Gujrati M, Lakka SS (2012) SPARC expression induces cell cycle arrest via STAT3 signaling pathway in medulloblastoma cells. Biochem Biophys Res Commun 417: 874-879.

31. Capper D, Mittelbronn M, Goeppert B, Meyermann R, Schittenhelm J (2010) Secreted protein, acidic and rich in cysteine (SPARC) expression in astrocytic tumour cells negatively correlates with proliferation, while vascular SPARC expression is associated with patient survival. Neuropathol Appl Neurobiol 36: 183-197.

32. Schultz C, Lemke N, Ge S, Golembieski WA, Rempel SA (2002) Secreted protein acidic and rich in cysteine promotes glioma invasion and delays tumor growth in vivo. Cancer Res 62: 6270-6277. 
Citation: Aljammal K, Ritz MF, Ramadoss A, Sauter G, Boulay JL, et al. (2015) Combined Expression of Nestin and SPARC Identifies In Situ Tumor Cells in Astrocytic Tumors of all Grades. J Cytol Histol 6: 313. doi:10.4172/2157-7099.1000313

Page 9 of 9

33. Turtoi A, Musmeci D, Naccarato AG, Scatena C, Ortenzi V, et al. (2012) Sparclike protein 1 is a new marker of human glioma progression. J Proteome Res 11: $5011-5021$.

34. Seno T, Harada H, Kohno S, Teraoka M, Inoue A, et al. (2009) Downregulation of SPARC expression inhibits cell migration and invasion in malignant gliomas. Int J Oncol 34: 707-715.
35. Ohgaki H, Kleihues $P$ (2013) The definition of primary and secondary glioblastoma. Clin Cancer Res 19: 764-772.

36. Auffinger B, Thaci B, Nigam P, Rincon E, Cheng Y, et al. (2012) New therapeutic approaches for malignant glioma: in search of the Rosetta stone. F1000 Med Rep 4: 18. 